Pacific Journal of Mathematics

TOPOLOGICAL SPACES DETERMINED BY LEFT IDEALS OF 


\title{
TOPOLOGICAL SPACES DETERMINED BY LEFT IDEALS OF SEMIGROUPS
}

\author{
Kenneth D. Magill, JR.
}

In this paper, we show how each (proper or improper) left ideal $Z$ of a semigroup $T$ determines, in a natural way, a topological space. The space will be denoted by $\mathscr{U}(T, Z)$ and will be referred to as the structure space of the pair $(T, Z)$. Any such structure space is compact and $T_{1}$, although it need not be Hausdorff. If $T$ contains a left identity, then the ideal $Z$ corresponds, in a natural way, to a subspace $\mathscr{R}(T, Z)$ of $\mathscr{U}(T, Z)$ which we refer to as the realization of $Z$. There is a homomorphism $\varphi$ from $T$ into $S(\mathscr{R}(T, Z)$ ) (for any space $X$, $S(X)$ denotes the semigroup, under composition, of all continuous functions mapping $X$ into $X$ ). Moreover, $\varphi$ is injective if and only if for every pair of distinct elements $a$ and $b$ of $T$, $a v \neq b v$ for some $v$ in $Z$.

If $T$ contains a left identity and, in addition, the pair $(T, Z)$ is admissible (Definition (1.6)), Then $\mathscr{U}(T, Z)$ is a Hausdorff compactification of $\mathscr{R}(T, Z)$ and each function in $\varphi[T]$ has a continuous extension to a function in $S(\mathscr{U}(T, Z))$. This results in a homomorphism from $T$ into $S(\mathscr{U}(T, Z))$ which, as before is injective if and only if for every pair of distinct elements $a$ and $b$ of $T, a v \neq b v$ for some $v$ in $Z$. These results are the applied to semigroups of continuous functions. For example, it is shown that if $X$ is a normal Hausdorff space which contains an arc and $Z(X)$ is the kernel (i.e., minimal two-sided ideal of $S(X))$, then the structure space of the pair $(S(X), Z(X))$ is the Stone-Čech compactification of $X$ and the realization of $Z(X)$ is homeomorphic to $X$.

1. The structure space of the pair $(T, Z)$.

Definition (1.1) Let $Z$ be a left ideal of a semigroup $T$. A nonempty subset $A$ of $T \times Z$ will be referred to as a bond if for any finite subset $\left\{\left(t_{i}, z_{i}\right)\right\}_{i=1}^{N} \subseteq A$, the system of equations $\left\{t_{i} x=z_{i}\right\}_{i=1}^{N}$ has a common solution $x$ in $Z$. A bond which is not properly contained in any other bond is referred to as an ultrabond. We denote the set of all ultrabonds by $\mathscr{U}(T, Z)$.

By the Lemma of Zorn, every bond is contained in an ultrabond. $T \times Z$ is a bond if and only if $Z=\{z\}$ for a right zero $z$ of $T$. In this case $\mathscr{U}(T, Z)=\{T \times Z\}$. For any $v \in Z$, the pairs $(t, t v), t \in T$, form a bond which we shall denote by $A_{v}$. 
Lemma (1.2). If the semigroup $T$ has a left identity, then $A_{v}$ is an ultrabond.

Proof. Let $e$ be a left identity of $T$ so that $(e, v) \in A_{v}$. If $(t, z)$ is not in $A_{v}$, i.e., $t v \neq z$, then the equations $t x=z$ and $e x=v$ have no common solution in $Z$.

Definition (1.3). For each pair, $(t, z) \in T \times Z$, we let

$$
H(t, z)=\{A \in \mathscr{U}(T, Z):(t, z) \in A\}
$$

The topological space which is obtained by taking $\{H(t, z):(t, z) \in T \times Z\}$ as a subbasis for the closed subsets of $\mathscr{U}(T, Z)$ is defined to be the structure space of the pair $(T, Z)$.

THEOREM (1.4). For a group $G, \mathscr{U}(G, G)$ consists of the ultrabonds $A_{v}, v \in G$. The closed subsets of $\mathscr{U}(G, G)$ are $\mathscr{U}(G, G)$ and the finite subsets of $\mathscr{U}(G, G)$.

Proof. Let $H(t, z)$ be given and let $v=t^{-1} z$. To prove the theorem, it will be sufficient to show that $H(t, z)=A_{v}$. Evidently, $A_{v} \in H(t, z)$. On the other hand, suppose $A_{u} \in H(t, z)$. Then $(t, z) \in A_{u}$ which implies that $(t, z)=(s, s u)$ for some $s \in T$. Thus $u=s^{-1} z=$ $t^{-1} z=v$ and $A_{u}=A_{v}$.

Theorem (1.5). Any structure space $\mathscr{U}(T, Z)$ is a compact $T_{1}$ space.

Proof. We first prove that $\mathscr{U}(T, Z)$ is $T_{1}$. Let $A$ and $B$ be distinct ultrabonds. Then $A-B$ is not empty and hence $A \in H(t, z)$, but $B \notin H(t, z)$, for some pair $(t, z)$. Thus $A=\cap\{H(t, z):(t, z) \in A\}$, and this is a closed set.

Now we prove that $\mathscr{U}(T, Z)$ is compact. By a result of Alexander $\{3$, p. 139 , Th. 6], the space $\mathscr{U}(T, Z)$ is compact if every family $\mathscr{F}=\left\{H\left(t_{\alpha}, z_{\alpha}\right): \alpha \in \Lambda\right\}$ of subbasic closed sets with the finite intersection property has a nonempty intersection. Let $A=\left\{\left(t_{\alpha}, z_{\alpha}\right): \alpha \in \Lambda\right\}$ and let $\left\{\left(t_{i}, z_{i}\right)\right\}_{i=1}^{N}$ be any finite subset of $A$. Then $\left\{H\left(t_{i}, z_{i}\right)\right\}_{i=1}^{N}$ is a finite subfamily of $\mathscr{F}$ and hence its intersection contains some ultrabond $B$. Thus $\left\{\left(t_{i}, z_{i}\right)\right\}_{i=1}^{N} \subseteq B$ and it follows that the system of equations $\left\{t_{i} x=z_{i}\right\}_{i=1}^{N}$ has a common solution $x$ in $Z$. This proves $A$ is a bond and it follows from Zorn's Lemma that $A$ is contained in some ultrabond $A^{*}$. Then $A^{*} \in \cap\left\{H\left(t_{\alpha}, z_{\alpha}\right): \alpha \in \Lambda\right\}$.

The complement of a set $X$ will be denoted by $\mathscr{C} X$.

Definition (1.6). The pair $(T, \mathrm{Z})$ is said to be admissible if $T$ is 
a semigroup, $Z$ is a left ideal of $T$ and the following condition is satisfied.

If $A$ is an ultrabond and $A \in \mathscr{C} H\left(t_{1}, z_{1}\right)$, there exist $\left(t_{2}, z_{2}\right)$ and $\left(t_{3}, z_{3}\right)$ in $T \times Z$ such that $A \in \mathscr{C} H\left(t_{2}, z_{2}\right)$ $\subseteq H\left(t_{3}, z_{3}\right) \subseteq \mathscr{C} H\left(t_{1}, z_{1}\right)$.

Although the structure space $\mathscr{C}(T, Z)$ of any pair $(T, Z)$ is $T_{1}$, it need not be Hausdorff. Indeed, it follows from Theorem (1.4) that $\mathscr{U}(G, G)$ is not Hausdorff if $G$ is an infinite group. If, however, the pair $(T, Z)$ is admissible, condition (1.6.1) implies that $\mathscr{U}(T, Z)$ is regular and a regular $T_{1}$ space is Hausdorff. This, together with Theorem (1.5) results in

THEOREM (1.7). The structure space $\mathscr{U}(T, Z)$ of any admissible pair $(T, Z)$ is a compact Hausdorff space.

Suppose the semigroup $T$ has a left identity. According to Lemma (1.2), $\left\{A_{v}: v \in Z\right\}$ is a subspace of $\mathscr{Q}(T, Z)$. This subspace will be denoted by $\mathscr{U}(T, Z)$ and will be referred to as the realization of $Z$. Every element $a$ in $T$ determines, in a very natural way, a mapping $\mathrm{f}_{a}$ from $\mathscr{R}(T, Z)$ into $\mathscr{R}(T, Z)$. The mapping $\mathrm{f}_{a}$ is defined by $\mathrm{f}_{a}\left(A_{v}\right)=$ $A_{a v}$. We are assured $a v \in Z$ since $Z$ is a left ideal of $T$. Before stating the next result, we mention again that for any space $X$, the symbol $S(X)$ denotes the semigroup, under composition, of all continuous functions mapping $X$ into $X$.

THEOREM (1.8). Let $T$ be a semigroup with a left identity and let $Z$ be a left ideal of $T$. Then for each element $a$ in $T$, the corresponding function $\mathrm{f}_{a}$ is a continuous mapping from $\mathscr{R}(T, Z)$ into $\mathscr{R}(T, Z)$. The mapping $\varphi$ from $T$ into $S(\mathscr{R}(T, Z)$ ) defined by $\varphi(a)=f_{a}$ is a homomorphism and is injective if and only if for each pair of distinct elements $a$ and $b$ of $T, a v \neq b v$ for some $v$ in $Z$.

Proof. The continuity of each $f_{a}$ follows from the equality

$$
\mathrm{f}_{a}^{-1}[\mathscr{R}(T, Z) \cap H(t, z)]=\mathscr{R}(T, Z) \cap H(t a, z)
$$

and the fact that $\{\mathscr{R}(T, Z) \cap H(t, z):(t, z) \in T \times Z\}$ is a subbasis for the closed subsets of $\mathscr{R}(T, Z)$.

Since $\mathrm{f}_{a} \circ \mathrm{f}_{b}=\mathfrak{f}_{a b}$ for all $a, b \in T$, the mapping $\varphi$ is a homomorphism from $T$ into $S(\mathscr{R}(T, Z)$ ). Now suppose $\phi$ is injective and $a$ and $b$ are distinct elements of $T$. Then $\mathrm{f}_{a}=\varphi(a) \neq \varphi(b)=\mathrm{f}_{b}$ and thus, $\mathrm{f}_{a}\left(A_{v}\right) \neq$ $\mathrm{f}_{b}\left(A_{v}\right)$ for some $v$ in $Z$. This implies $A_{a v} \neq A_{b v}$ and hence $a b \neq b v$. On the other hand, suppose that for any distinct pair of elements $a$, 
$b \in T, a v \neq b v$ for some $v$ in $Z$. Then $A_{a v} \neq A_{b v}$ since $(e, a v) \in A_{a v}-A_{b v}$ (where $e$ denotes a left identity of $T$ ). Thus, $\mathrm{f}_{a}\left(A_{v}\right) \neq \mathrm{f}_{b}\left(A_{v}\right)$ and it follows that $\phi$ is injective.

For the proof of the next theorem, it will be convenient to have the following

Lemma (1.9). Suppose $T$ is a semigroup with a left identity and $Z$ is a left ideal of $T$. If

$$
\mathscr{U}(T, Z)=H\left(t_{1}, z_{1}\right) \cup H\left(t_{2}, z_{2}\right)
$$

for $\left(t_{1}, z_{1}\right)$ and $\left(t_{2}, z_{2}\right)$ in $T \times Z$, then, for any $a$ in $T$,

$$
\mathscr{U}(T, Z)=H\left(t_{1} a, z_{1}\right) \cup H\left(t_{2} a, z_{2}\right) .
$$

Proof. Since $T$ has a left identity, each $A_{v}, v \in Z$, is an ultrabond and we have

$$
\mathscr{R}(T, Z) \subseteq H\left(t_{1}, z_{1}\right) \cup H\left(t_{2}, z_{2}\right) .
$$

This implies that for any $a$ in $T$,

$$
\mathscr{R}(T, Z) \subseteq H\left(t_{1} a, z_{1}\right) \cup H\left(t_{2} a, z_{2}\right) \text {. }
$$

To see this, let $A_{v}$ in $\mathscr{R}(T, Z)$ be given. Then by (1.9.1), either $A_{a v} \in H\left(t_{1}, z_{1}\right)$ or $A_{a v} \in H\left(t_{2}, z_{2}\right)$. Suppose the former holds. Then $\left(t_{1}\right.$, $\left.z_{1}\right) \in A_{a v}$ which implies $\left(t_{1}, z_{1}\right)=(s, s a v)$ for some $s$ in $T$. Thus $t_{1}=s$ and $z_{1}=s a v=t_{1} a v$ and it follows that $\left(t_{1} a, z_{1}\right)=\left(t_{1} a, t_{1} a v\right) \in A_{v}$, i.e., $A_{v}, \in H\left(t_{1} a, z_{1}\right)$.

Now suppose some ultrabond $B$ does not belong to either $H\left(t_{1} a, z_{1}\right)$ or $H\left(t_{2} a, z_{2}\right)$. Then neither $\left(t_{1} a, z_{1}\right)$ nor $\left(t_{2} a, z_{2}\right)$ belong to $B$ and it follows that there exist two finite subfamilies $\left.\left\{\left(k_{i}, v_{i}\right)\right)\right\}_{i=1}^{N}\left\{\left(s_{i}, y_{i}\right)\right\}_{i=1}^{M}$ of $B$ such that no solution from $Z$ of the system $\left\{k_{i} x=v_{i}\right\}_{i=1}^{N}$ satisfies the equation $\left(t_{1} a\right) x=z_{1}$ and, similarly, no solution from $Z$ of the system $\left\{s_{i} x=y_{i}\right\}_{i=1}^{M}$ satisfies the equation $\left(t_{2} a\right) x=z_{2}$. Since $B$ is an ultrabond, there exists a solution $b$ in $Z$ to the system $\left\{k_{i} x=v_{i}\right\}_{i=1}^{N} \cup\left\{s_{i} x=y_{i}\right\}_{i=1}^{M}$. But then, $\left(t_{1} a\right) b \neq z_{1}$ and $\left(t_{2} a\right) b \neq z_{2}$ which implies $\left(t_{1} a, z_{1}\right) \notin A_{b}$ and $\left(t_{2} a, z_{2}\right) \notin A_{b}$. This, however, is equivalent to the statement $A_{b} \notin H\left(t_{1} a, z_{1}\right) \cup H\left(t_{2} a, z_{2}\right)$ which contradicts statement (1.9.2). Therefore, the ultrabond $B$ must belong to either $H\left(t_{1} a, z_{1}\right)$ or $H\left(t_{2} a, z_{2}\right)$.

THEOREM (1.10). Suppose the pair $(T, Z)$ is admissible and $T$ has a left identity. Then $\mathscr{U}(T, Z)$ is a Hausdorff compactification of $\mathscr{R}(T, Z)$. Moreover, for each a in $T$, the function $\mathrm{f}_{a}$ in $S(\mathscr{R}(T, Z)$ ) has a unique extention to a function $\mathrm{f}_{a}^{E}$ in $S(\mathscr{U}(T, Z))$. The mapping 
$\psi$ from $T$ into $S(\mathscr{Q}(T, Z))$ which is defined by $\psi(a)=\mathfrak{f}_{a}^{E}$ is a homomorphism and is injective if and only if for each pair of distinct elements $a$ and $b$ of $T, a v \neq b v$ for some $v$ in $Z$.

Proof. The space $\mathscr{C}(T, Z)$ is a compact Hausdorff space by Theorem (1.7). Now suppose $\mathscr{U}(T, Z) \neq \cup\left\{H\left(t_{i}, z_{i}\right)\right\}_{i=1}^{N}$. Then some ultrabond $A$ does not contain any of the pairs $\left(t_{i}, z_{i}\right)$. This implies that for each $i$, there exists a finite subfamily $\left\{\left(s_{i j}, y_{i j}\right)\right\}_{j=1}^{N i}$ of $A$ for which the system of equations,

$$
t_{i} x=z_{i}, s_{i 1} x=z_{i 1}, s_{i 2} x=z_{i 2}, \cdots, s_{i_{i}} x=z_{i_{i}},
$$

has no common solution $X$ in $Z$. However, there does exist an element $v$ in $Z$ such that $\mathrm{s}_{i j} v=z_{i j}$ for each $i$ and $j$. It then follows from the previous statement that $t_{i} v \neq z_{i}$ for each $i$. Thus

$$
A_{v} \notin \cup\left\{H\left(t_{i}, z_{i}\right)\right\}_{i=1}^{N} \text {. }
$$

This proves that $\mathscr{R}(T, Z)$ is a dense subset of $\mathscr{C}(T, Z)$. Therefore, $\mathscr{U}(T, Z)$ is indeed a Hausdorff compactification of $\mathscr{R}(T, Z)$.

By Theorem (1.8), each function $\mathrm{f}_{a}, a \in T$, is a continuous mapping prom $\mathscr{R}(T, Z)$ into $\mathscr{R}(T, Z)$. We define a mapping $\mathfrak{f}_{a}^{E}$ from $\mathscr{U}(T, Z)$ into $\mathscr{C}(T, Z)$ as follows: for any ultrabond $A$, let

$$
\langle A\rangle=\{(t, z) \in T \times Z:(t a, z) \in A\} .
$$

Either $T \times Z=A$ (in which case $\langle A\rangle \neq \varnothing$ ) or some $\left(t_{1}, z_{1}\right)$ does not belong to $A$. It then follows from (1.6.1) that there exist $\left(t_{2}, z_{2}\right)$ and $\left(t_{3}, z_{3}\right)$ in $T \times Z$ such that $\mathscr{C} H\left(t_{2}, z_{2}\right) \subseteq H\left(t_{3}, z_{3}\right)$. Thus $\mathscr{C}(T, Z)=$ $H\left(t_{2}, z_{2}\right) \cup H\left(t_{3}, z_{3}\right)$ and the previous lemma implies

$$
\mathscr{Q}(T, Z)=H\left(t_{2} a, z_{2}\right) \cup H\left(t_{3} a, z_{3}\right) .
$$

Hence $A$ contains either $\left(t_{2} a, z_{2}\right)$ or $\left(t_{3} a, z_{3}\right)$ and this implies that $\langle A\rangle$ contains either $\left(t_{2}, z_{2}\right)$ or $\left(t_{3}, z_{3}\right)$. This proves that $\langle A\rangle \neq \varnothing$. For any finite subset $\left\{\left(t_{i}, z_{i}\right)\right\}_{i=1}^{N} \subseteq\langle A\rangle,\left\{\left(t_{i} a, z_{i}\right)\right\}_{i=1}^{N} \subseteq A$ which implies that the system of equations $\left\{\left(t_{i} a\right) x=z_{i}\right\}_{i=1}^{N}$ has a solution $v$ in $Z$. Since $Z$ is a left ideal, $a v \in Z$ and $a v$ is a solution of the system $\left\{t_{i} x=z_{i}\right\}_{i=1}^{N}$. This proves that $\langle A\rangle$ is a bond. By Zorn's Lemma, $\langle A\rangle$ is contained in an ultrabond $\langle A\rangle^{*}$ and we define $\mathrm{f}_{a}^{E}(A)=\langle A\rangle^{*}$. For the present, it may seem as though $\mathrm{f}_{a}^{E}$ is not unique ( $A$ might conceivably be contained in more than one ultrabond), but we shall eventually see that this is not the case. Let us note that

$$
\left\{(t, z) \in T \times Z:(t a, z) \in A_{v}\right\}=A_{a v} .
$$

Since $A_{a v}$ is an ultrabond, $\left\langle A_{v}\right\rangle^{*}=A_{a v}$. Thus, for any $v$ in $Z$, 


$$
\mathfrak{f}_{a}^{E}\left(A_{v}\right)=\left\langle A_{v}\right\rangle^{*}=A_{a v}=\mathfrak{f}_{a}\left(A_{v}\right)
$$

which proves that $f_{a}^{E}$ is an extension of $f_{a}$.

We now establish the continuity of $f_{a}^{E}$. Suppose

$$
\mathfrak{f}_{a}^{E}(A)=\langle A\rangle^{*} \in \mathscr{C} H\left(t_{1}, z_{1}\right) \text {. }
$$

By condition (1.6.1), there exist $\left(t_{2}, z_{2}\right)$ and $\left(t_{3}, z_{3}\right)$ in $T \times Z$ such that

$$
\langle A\rangle^{*} \in \mathscr{C} H\left(t_{2}, z_{2}\right) \subseteq H\left(t_{3}, z_{3}\right) \subseteq \mathscr{C} H\left(t_{1}, z_{1}\right) \text {. }
$$

Suppose $A \in H\left(t_{2} a, z_{2}\right)$. Then $\left(t_{2}, z_{2}\right) \in\langle\mathrm{A}\rangle$ which implies $\langle A\rangle^{*} \in H\left(t_{2}, z_{2}\right)$. This, of course, is a contradiction so we conclude $A \in \mathscr{C} H\left(t_{2} a, z_{2}\right)$. Now we assert

$$
\mathfrak{f}_{a}^{E}\left[\mathscr{C} H\left(t_{2} a, z_{2}\right)\right] \subseteq \mathscr{C} H\left(t_{1}, z_{1}\right) .
$$

Suppose $B \in \mathscr{C} H\left(t_{2} a, z_{2}\right)$ and $f_{a}^{E}(B)=\langle B\rangle^{*} \in H\left(t_{2}, z_{2}\right)$. Now

$$
H\left(t_{2}, z_{2}\right) \cup H\left(t_{3}, z_{3}\right)=\mathscr{U}(T, Z) .
$$

This, in conjection with Lemma (1.9), implies $H\left(t_{2} a, z_{2}\right)=\mathscr{C}(T, Z)$. Since $B \notin H\left(t_{2} a, z_{2}\right)$, it follows that $B \in H\left(t_{3} a, z_{3}\right)$. Thus, $\left(t_{3} a, z_{3}\right) \in B$ which implies $\left(t_{3}, z_{3}\right) \in\langle B\rangle \subseteq\langle B\rangle^{*}$. This, in turn, implies that $\mathrm{f}_{a}^{E}(B) \in H\left(t_{3}, z_{3}\right)$. We have shown that either $\mathfrak{f}_{a}^{E}(B) \in \mathscr{C} H\left(t_{2}, z_{2}\right)$ or $\mathrm{f}_{a}^{E}(B) \in H\left(t_{3}, z_{3}\right)$. In either event, $\mathrm{f}_{a}^{E}(B) \in \mathscr{C} H\left(t_{1}, z_{1}\right)$ and (1.10.1) has been verified. Now, $\{\mathscr{C} H(t, z):(t, z) \in T \times Z\}$ is a subbasis for the open subsets of $\mathscr{U}(T, Z)$. Property (1.10.1) implies that the preimage, under $\mathrm{f}_{a}^{E}$, of each of these subbasic open sets is open. Hence the preimage of every open set is open and the continuity of $f_{a}^{E}$ has been established. The uniqueness of the extension $f_{a}^{E}$ is a consequence of the well known fact that if any two continuous functions from an arbitrary space into a Hausdorff space agree on a dense subset, then the functions are identical.

For any two elements $a$ and $b$ of $T$, the functions $\mathrm{f}_{a b}^{E}$ and $\mathrm{f}_{a}^{E} \circ \mathrm{f}_{b}^{E}$ agree on the dense subset $\mathscr{R}(T, Z)$ of $\mathscr{U}(T, Z)$. Thus $\mathfrak{f}_{a b}^{E}=\mathfrak{f}_{a}^{E} \circ f_{b}^{E}$ and it follows that the mapping $\psi$ defined by $\psi(a)=\mathfrak{f}_{a}^{E}$ is a homomorphism from $T$ into $S(\mathscr{U}(T, Z)$ ). Finally, $\psi$ is an injection if and only if the homomorphism $\varphi$ in the statement of Theorem (1.8) is an injection. Therefore, $\psi$ is an injection if and only if for each pair of distinct elements $a$ and $b$ of $T, a v \neq b v$ for some $v$ in $Z$.

2. Applications to semigroups of continuous functions. Now we consider structure spaces of pairs of the form $(S(X), Z(X))$ where, as before, $S(X)$ denotes the semigroup (under composition) of all continuous functions mapping $X$ into $X$ and $Z(X)$ denotes the kernel (i.e., minimal two-sided ideal) of $S(X)$. The kernel, in this case, is 
simply the set of all constant functions. We denote the constant function determined by the point $z$ in $X$ by $z$, i.e., $z(p)=z$ for all $p$ in $X$. Thus, for the pair $(S(X), Z(X))$, bonds consist of ordered pairs of the form $(f, z)$ where $f \in S(X)$ and $z$ is a constant function. To make the notation less complicated, we will denote the structure space of the pair $(S(X), Z(X))$ simply by $\mathscr{C}(X)$ rather than by $\mathscr{C}(S(X), Z(X))$. Similarly, the realization of $Z(X)$ will be denoted by $\mathscr{R}(X)$ rather than by $\mathscr{R}(S(X), Z(X))$

In the sequel, we will use the following facts without explicit mention.

(i) For $f$ in $S(X)$ and $\boldsymbol{y}, \boldsymbol{z}$ in $Z(X)$, the statement $f \circ \boldsymbol{y}=\boldsymbol{z}$ is equivalent to the statement $f(y)=z$.

(ii) A subset $A$ of $S(X) \times Z(X)$ is a bond if and only if $\left\{f^{-1}\{z\}\right.$ : $(f, z) \in A\}$ has the finite intersection property.

(iii) A bond is an ultrabond if and only if $(f, z) \notin A$ implies $f^{-1}\{z\} \cap g_{1}^{-1}\left\{y_{1}\right\} \cap \cdots \cap g_{N}^{-1}\left\{y_{N}\right\}=\varnothing$ for some finite subfamily $\left\{\left(g_{i}, \boldsymbol{y}_{i}\right)\right\}_{i=1}^{N}$ of $A$.

Definition (2.1). We reserve the symbol e to denote the mapping from $X$ onto $\mathscr{R}(X)$ which is defined by $\mathrm{e}(X)=A_{x}$. The mapping $\mathrm{e}$ will be referred to as the canonical mapping from $X$ onto $\mathscr{R}(X)$.

Definition (2.2). A topological space $X$ is an $S^{*}$-space if it is $T_{1}$ and for each closed subset $F$ of $X$ and each point $p \in X-F$, there exists a function $f$ in $S(X)$ and a point $y$ in $X$ such that $f(x)=y$ for each $x$ in $F$ and $f(p) \neq y$.

This class of spaces was introduced in [5] and, as pointed out there, includes all completely regular Hausdorff spaces which contain an arc as well as all 0-dimensional Hausdorff spaces.

Theorem (2.3). Suppose $X$ is an $S^{*}$-space. Then the canonical mapping e from $X$ onto $\mathscr{R}(X)$ is a homeomorphism. In this case, the homomorphism $\varphi$ from $S(X)$ into $S(\mathscr{R}(X))$ defined in Theorem (1.8) is actually an isomorphism onto $S(\mathscr{R}(X))$. Moreover, this isomorphism is given by $\varphi(f)=\mathfrak{e} \circ f \circ e^{-1}$ for each $f$ in $S(X)$.

Proof. Let $F$ be a closed subset of $X$. For each $p \in X-F$, there exists a function $f_{p} \in S(X)$ and a point $y_{p} \in X$ such that $f_{p}(x)=y_{p}$ for $x$ in $F$ and $f_{p}(p) \neq y_{p}$. Thus,

$$
F=\cap\left\{f_{p}^{-1}\left\{y_{p}\right\}: p \in X-F\right\}
$$

and it follows that $\left\{f^{-1}\{y\}: f \in S(X), y \in X\right\}$ is a subbasis for the closed subsets of $X$. The fact that $\mathrm{e}$ is a homeomorphism from $X$ onto $\mathscr{R}(X)$ is a consequence of this and the fact that 


$$
\mathrm{e}^{-1}[\mathscr{R}(X) \cap H(f, \boldsymbol{y})]=f^{-1}\{y\}
$$

and

$$
\mathrm{e}\left[f^{-1}\{y\}\right]=\mathscr{R}(X) \cap H(f, \boldsymbol{y})
$$

for each $(f, \boldsymbol{y})$ in $S(X) \times Z(X)$.

Now, for any $A_{v} \in \mathscr{R}(X)$ and any $f$ in $S(X)$,

$$
\mathfrak{e} \circ f \circ \mathrm{e}^{-1}\left(A_{v}\right)=\mathrm{e}(f(v))=A_{f(v)}=\mathfrak{f}_{f}\left(A_{v}\right)=\varphi(f)\left(A_{v}\right) .
$$

Thus, $\phi(f)=e \circ f \circ e^{-1}$ for each $f$ in $S(X)$. This implies that $\phi$ is an isomorphism from $S(X)$ onto $S(\mathscr{R}(X))$ since $\mathrm{e}$ is a homeomorphism from $X$ onto $R(X)$.

It follows easily that if $X$ and $Y$ are homeomorphic, then $S(X)$ and $S(Y)$ are isomorphic. As one might expect, however, the converse is not true. An easy counter-example is obtained by letting $X$ be a discrete space with more than one element and letting $Y$ be the same set, but endowed with the indiscrete topology. The spaces $X$ and $Y$ are certainly not homeomorphic but the identity mapping is an isomorphism from $S(X)$ onto $S(Y)$. For $S^{*}$-spaces, however, the converse does hold. Let us first observe that the algebraic structure of a semigroup $T$ containing a left identity completely determines the topological structure of $\mathscr{R}\left(T, Z_{T}\right)$ where $Z_{T}$ denotes the kernel of $T$. That is, $\mathscr{R}\left(T, Z_{T}\right)$ and $\mathscr{R}\left(W, Z_{W}\right)$ are homeomorphic if $T$ and $W$ are isomorphic. Therefore, if $X$ and $Y$ are any two topological spaces such that $S(X)$ and $S(Y)$ are isomorphic, then $\mathscr{R}(X)$ and $\mathscr{R}(Y)$ are homeomorphic. If, in addition, $X$ and $Y$ are $S^{*}$-spaces, it follows from Theorem (2.3) that $X$ is homeomorphic to $\mathscr{R}(X)$ and $Y$ is homeomorphic to $\mathscr{R}(X)$. These observations result in

Corollary (2.4). Two $S^{*}$-spaces $X$ and $Y$ are homeomorphic if and only if $S(X)$ and $S(Y)$ are isomorphic.

This result is also an immediate consequence of Theorem 1 of [5]. Now we seek a class of spaces with the property that for any space $X$ within the class, the pair $(S(X), Z(X))$ is admissible. The class of $S^{*}$-spaces does not have this property. To see this, let $X$ be any infinite set and topologize $X$ by letting $X$ and its finite subsets be the closed subsets of $X$. Let $F$ be any closed subset of $X$ and let $p$ be any point in the complement of $F$. Choose $q \neq p$ and define a function $f$ mapping $X$ into $X$ by

$$
f(x)=q \text { for } x \text { in } F
$$

and 


$$
f(x)=x \text { for } x \text { in } X-F .
$$

The function $f$ is continuous since preimages of finite sets are finite. Since $X$ is a $T_{1}$ space, it follows that $X$ is an $S^{*}$-space. Therefore, Theorem (2.3) implies that $X$ is homeomorphic to $\mathscr{R}(X)$ which, in turn, implies that $\mathscr{R}(X)$ is not Hausdorff. From this it follows that the pair $(S(X), Z(X))$ cannot be admissible since otherwise Theorem (1.10) would imply that $\mathscr{R}(X)$ is Hausdorff.

At this point, we do not know if $(S(X), Z(X))$ must be admissible when $X$ is a Hausdorff $S^{*}$-space. However, we are able to find a rather extensive subclass of the class of Hausdorff $S^{*}$-spaces with the property that $(S(X), Z(X))$ is admissible for any $X$ from this subclass. We refer to these sapces as strong $S^{*}$-spaces and we define them as follows:

Definition (2.5). A space $X$ is a strong $S^{*}$-space if it if Hausdorff and for every pair of disjoint closed subsets $H$ and $K$ of $X$, there exist distinct points $p$ and $q$ of $X$ and a function $f$ in $S(X)$ such that $f(x)=p$ for $x$ in $H$ and $f(x)=q$ for $x$ in $K$.

Theorem (2.6). The class of strong $S^{*}$-spaces includes all normal Hausdorff spaces which contain an arc as well as all those Hausdorff spaces with the property that for any open subset $G$ containing a closed subset $K$, there exists an open-and-closed subset $H$ such that $K \subseteq H \subseteq G$.

Proof. First suppose $X$ is normal and there exists a homeomorphism $h$ from the closed unit interval $I$ into $X$. For any two disjoint closed subsets $H$ and $K$ of $X$ there exists, by Urysohn's Lemma, a continuous function $f$ mapping $X$ into $I$ such that $f(x)=0$ for $x$ in $H$ and $f(x)=1$ for $x$ in $K$. The function $h \circ f$ satisfies the conditions set forth in Definition (2.5).

Now suppose $X$ has the property that for every closed subset $K$ and every open subset $G$ such that $K \cong G$, there exists an-open-and closed subset $H$ such that $K \subseteq H \subseteq G$. Then for any two disjoint closed subsets $K$ and $W$ of $X$, there exists an open-and-closed subset $H$ such that $K \subseteq H \subseteq X-W$. Choose two distinct points $p$ and $q$ of $X$. If this cannot be done, it is immediate that $X$ is a strong $S^{*}$-space. The function $f$ defined by $f(x)=p$ for $x$ in $H$ and $f(x)=q$ for $x$ in $X-H$ has the properties required in Definition (2.5) and it follows that $X$ is a strong $S^{*}$-space.

It follows rather easily that every strong $S^{*}$-space is a normal Hausdorff space. The converse, however, is not true and we will later 
discuss some examples which verify this.

THEOREM (2.7). If $X$ is a strong $S^{*}$-space, then the pair $(S(X)$, $Z(X))$ is admissible. Furthermore, if $X$ contains an arc, then the structure space $\mathscr{U}(X)$ of the pair $(S(X), Z(X))$ is the Stone-ČCech compactification of $X$.

Proof. Suppose $A$ is an ultrabond and $A \in \mathscr{C} H(f, z)$. Then for some finite subfamily $\left\{\left(g_{i}, y_{i}\right)\right\}_{i=1}^{N}$ of $A, f^{-1}\{z\} \cap W \neq \varnothing$ where $W=$ $\cap\left\{g_{i}^{-1}\left\{y_{i}\right\}\right\}_{i=1}^{N}$. Then $W \subseteq \mathscr{C}\left[f^{-1}\{z\}\right]$ and since $X$ is normal, there exists an open subset $G$ of $X$ and a closed subset $K$ of $X$ such that

$$
W \cong G \subseteq K \subseteq \mathscr{C}\left[f^{-1}\{z\}\right] .
$$

Since $X$ is a strong $S^{*}$-space, there exist two functions $h$ and $k$ in $S(X)$ and points $a, b, p, q$ in $X$ with $a \neq b$ and $p \neq q$ such that

$$
\begin{aligned}
& h(x)=a \text { for } x \text { in } X-G \\
& h(x)=b \text { for } x \text { in } W \\
& k(x)=p \text { for } x \text { in } K \\
& k(x)=q \text { for } x \text { in } f^{-1}\{z\} .
\end{aligned}
$$

Then,

$$
W \cong \mathscr{C}\left[h^{-1}\{a\}\right] \subseteq k^{-1}\{p\} \subseteq \mathscr{C}\left[f^{-1}\{z\}\right]
$$

This implies,

$$
A \in \mathscr{C} H(h, \boldsymbol{a}) \subseteq H(k, \boldsymbol{p}) \subseteq \mathscr{C} H(f, \boldsymbol{z})
$$

and hence, the pair $(S(X), Z(X))$ is admissible.

Since every strong $S^{*}$-space is an $S^{*}$-space, it follows from Theorem (2.3) that the canonical mapping e from $X$ onto $\mathscr{R}(X)$ is a homeomorphism. It now follows from Theorem (1.10) that $\mathscr{U}(X)$ (or more precisely, the pair $(\mathscr{U}(X), e))$ is a Hausdorff compactification of $X$. To show that $\mathscr{U}(X)$ is the Stone-Čech compactification of $X$, it is sufficient to show that any continuous function mapping $\mathscr{R}(X)$ into the closed unit interval $I$ can be continuously extended over $\mathscr{U}(X)$. Let $f$ be any continuous function from $\mathscr{R}(X)$ into $I$. Since $X$ contains an arc, $\mathscr{R}(X)$ does also and there exists a homeomorphism $g$ from $I$ into $\mathscr{R}(X)$. Thus $g \circ f$ belongs to $S(\mathscr{R}(X))$. According to Theorem (2.3), $g \circ f=\varphi(h)=\mathrm{f}_{h}$ for some $h$ in $S(X)$ and by Theorem (1.10), $\mathrm{f}_{h}$ has a continuous extension $\mathrm{f}_{h}^{E}$ which maps $\mathscr{U}(X)$ into $\mathscr{U}(X)$. Now

$$
\begin{aligned}
\mathrm{f}_{h}^{E}[\mathscr{U}(X)] & =\mathrm{f}_{h}^{E}[\operatorname{cl}[\mathscr{R}(X)]] \subseteq \operatorname{cl}\left[\mathrm{f}_{h}^{E}[\mathscr{R}(X)]\right] \\
& =\operatorname{cl}[g[f[\mathscr{R}(X)]]] \leqq \operatorname{cl}[g[I]]=g[I] .
\end{aligned}
$$


Thus, the image of $f_{h}^{E}$ is a subset of the range of $g$ and it follows that $g^{-1} \circ f_{h}^{E}$ is a continuous mapping from $\mathscr{U}(X)$ into $I$. Moreover, for any $A$ in $\mathscr{R}(X)$,

$$
\left(g^{-1} \circ \mathfrak{f}_{h}^{E}\right)(A)=g^{-1}\left(\mathfrak{f}_{h}(A)\right)=g^{-1}(g(f(A))=f(A)
$$

and $g^{-1} \circ \mathfrak{f}_{h}^{E}$ is the desired extension of $f$.

The following corollary is an immediate consequence of Theorems (2.6) and (2.7).

COROLLARY (2.8). If $X$ is a normal Hausdorff space which contains an arc, then the structure space of the pair $(S(X), Z(X))$ is the Stone- $\breve{C} e c h$ compactification of $X$.

3. Some examples and concluding remarks. De Groot, in [2] has proven the existence of a class of spaces which, for the study of semigroups of continuous functions, has turned out to be a source of very illuminating examples. He has shown (Th. 3, p. 87) that there exists a family $\mathscr{G}$ consisting of $2^{c} 1$-dimensional connected and locally connected subspaces of the Euclidean plane such that for any $X \in \mathscr{G}$, the only continuous functions mapping $X$ into itself are the constant functions and the identity function. Furthermore, for any two distinct spaces $X$ and $Y$ of $\mathscr{G}$, the only continuous functions mapping $X$ into $Y$ are the constant functions. It follows that for any $X$ in $\mathscr{G}, S(X)$ is a left zero semigroup with identity, i.e., the product of any two elements ( $\neq$ identity) is the element on the left. Since each such $X$ consists of more than one point and is connected, its cardinality must be $c$. This implies that the cardinality of each $S(X)$ is also $c$. Therefore, for any two spaces $X$ and $Y$ of $\mathscr{C}$, any bijection from $S(X)$ onto $S(Y)$ which takes the identity of the former onto the identity of the latter is an isomorphism. However, $X$ and $Y$ are not homeomorphic if $X \neq Y$. Of course, none of the spaces in $\mathscr{G}$ are $S^{*}$-spaces and therefore none can be strong $S^{*}$-spaces. This verifies the assertion following Theorem (2.6) that a normal Hausdorff space need not be a strong $S^{*}$-space.

Corollary (2.8) states that one can obtain $\beta X$ from the semigroup $S(X)$ if $X$ is normal, Hausdorff and contains an arc. Specifically, for such a space $X$, the structure space of $(S(X), Z(X))$ is the Stone-Čech compactification, $\beta X$, of $X$. The properties of being normal and Hausdorff are not sufficient for this, even when coupled with connectedness and local connectedness. All the spaces in $\mathscr{G}$ satisfy these properties, yet for no space $X$ in $\mathscr{G}$ is the structure space $\mathscr{U}(X)=\beta X$. Since all the $S(X), X \in \mathscr{G}$, are isomorphic, all the $\mathscr{U}(X), X \in \mathscr{G}$, are homeomorphic. We shall determine the structure of one (and hence, 
all) $\mathscr{U}(X)$. Let $i$ denote the identity function on $X$. For any $z$ in $Z(X)$,

$$
A_{\boldsymbol{z}}=\{(\boldsymbol{y}, \boldsymbol{y}): \boldsymbol{y} \in Z(X)\} \cup\{(i, \boldsymbol{z})\} .
$$

Each $A_{z}$ is an ultrabond and, conversely, each ultrabond is of the form $A_{z}$ for some $z$ in $Z(X)$. Then, for any $y, z$ in $Z(X)$,

$$
H(\boldsymbol{y}, \boldsymbol{z})=\varnothing \text { if } y \neq z
$$

and

$$
H(\boldsymbol{y}, \boldsymbol{y})=\left\{A_{\boldsymbol{v}}: \boldsymbol{v} \in Z(X)\right\}
$$

Furthermore,

$$
H(i, z)=\left\{A_{z}\right\}
$$

From these considerations, it follows that $\mathscr{U}(X)=\mathscr{R}(X)$ and that the closed subsets of $\mathscr{U}(X)$ are $\mathscr{U}(X)$ and the finite subsets of $\mathscr{U}(X)$. Thus, $\mathscr{U}(X)$ is not Hausdorff and, consequently, cannot be $\beta X$. Furthermore, it follows from Theorem (1.10) that the pair $(S(X), Z(X))$ is not admissible.

At this point it seems appropriate to make an observation concerning the conclusions of Theorems (2.3) and (2.7). Essentially, we proved that these conclusions are valid when we restrict ourselves to spaces with an ample supply of continuous functions. The previous discussion indicates that some such restrictions are necessary.

In conclusion, we take the opportunity to express our appreciation to the referee for his suggestions which have improved the presentation of this paper.

\section{BIBLIOGRAPHY}

1. A. H. Clifford and G. B. Preston, The Algebraic Theory of Semigroups, Mathematical Surveys, Number 7.

2. J. de Groot, Groups represented by homeomorphism groups, I, Math. Annalen 138 (1959), 80-102.

3. J. L. Kelley, General Topology, Van Nostrand, Princeton, 1955.

4. E. S. Ljapin, Semigroups, Translations of Mathematical Monographs, Vol. 3, Amer. Math. Soc., 1963.

5. K. D. Magill, Jr., Another S-admissible class of spaces, Proc. Amer. Math. Soc. 18 (1967), 295-298.

Received June 22, 1966.

State University of New York at Buffalo 


\title{
PACIFIC JOURNAL OF MATHEMATICS
}

\author{
EDITORS
}

\section{H. ROYDEN}

Stanford University

Stanford, California

\section{J. P. JANS}

University of Washington Seattle, Washington 98105

\section{J. DugundJI}

Department of Mathematics

Rice University

Houston, Texas 77001

RICHARD ARENS

University of California

Los Angeles, California 90024

\section{ASSOCIATE EDITORS}

E. F. BECKENBACH
F. WOLF

K. YosidA

\section{SUPPORTING INSTITUTIONS}

\author{
UNIVERSITY OF BRITISH COLUMBIA \\ CALIFORNIA INSTITUTE OF TECHNOLOGY \\ UNIVERSITY OF CALIFORNIA \\ MONTANA STATE UNIVERSITY \\ UNIVERSITY OF NEVADA \\ NEW MEXICO STATE UNIVERSITY \\ OREGON STATE UNIVERSITY \\ UNIVERSITY OF OREGON \\ OSAKA UNIVERSITY \\ UNIVERSITY OF SOUTHERN CALIFORNIA
}

\author{
STANFORD UNIVERSITY \\ UNIVERSITY OF TOKYO \\ UNIVERSITY OF UTAH \\ WASHINGTON STATE UNIVERSITY \\ UNIVERSITY OF WASHINGTON \\ MERICAN MATHEMATICAL SOCIETY \\ CHEVRON RESEARCH CORPORATION \\ TRW SYSTEMS \\ NAVAL ORDNANCE TEST STATION
}

Mathematical papers intended for publication in the Pacific Journal of Mathematics should be in typed form or offset-reproduced, double spaced with large margins. Underline Greek letters in red, German in green, and script in blue. The first paragraph or two must be capable of being used separately as a synopsis of the entire paper. It should not contain references to the bibliography. Manuscripts may be sent to any one of the four editors. All other communications to the editors should be addressed to the managing editor, Richard Arens, University of California, Los Angeles, California 90024.

Each author of each article receives 50 reprints free of charge; additional copies may be obtained at cost in multiples of 50 .

The Pacific Journal of Mathematics is published monthly. Effective with Volume 16 the price per volume (3 numbers) is $\$ 8.00$; single issues, $\$ 3.00$. Special price for current issues to individual faculty members of supporting institutions and to individual members of the American Mathematical Society: $\$ 4.00$ per volume; single issues $\$ 1.50$. Back numbers are available.

Subscriptions, orders for back numbers, and changes of address should be sent to Pacific Journal of Mathematics, 103 Highland Boulevard, Berkeley 8, California.

Printed at Kokusai Bunken Insatsusha (International Academic Printing Co., Ltd.), 7-17, Fujimi 2-chome, Chiyoda-ku, Tokyo, Japan.

PUBLISHED BY PACIFIC JOURNAL OF MATHEMATICS, A NON-PROFIT CORPORATION

The Supporting Institutions listed above contribute to the cost of publication of this Journal, but they are not owners of publishers and have no responsibility for its content or policies. 


\section{Pacific Journal of Mathematics}

\section{Vol. 24, No. $2 \quad$ June, 1968}

John Suemper Alin and Spencer Ernest Dickson, Goldie's torsion theory and its derived functor . . . . . . . . . . . . . . . . . . . . . . . . . . . 195

Steve Armentrout, Lloyd Lesley Lininger and Donald Vern Meyer,

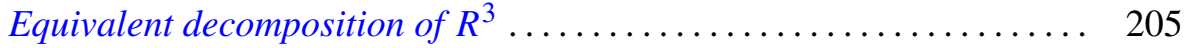

James Harvey Carruth, A note on partially ordered compacta ........... 229

Charles E. Clark and Carl Eberhart, A characterization of compact

connected planar lattices........................... 233

Allan Clark and Larry Smith, The rational homotopy of a wedge ......... 241

Donald Brooks Coleman, Semigroup algebras that are group algebras .... 247

John Eric Gilbert, Convolution operators on $L^{p}(G)$ and properties of

locally compact groups ............................. 257

Fletcher Gross, Groups admitting a fixed-point-free automorphism of order

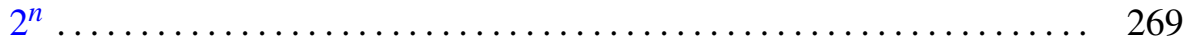

Jack Hardy and Howard E. Lacey, Extensions of regular Borel measures . . . 277

R. G. Huffstutler and Frederick Max Stein, The approximation solution of

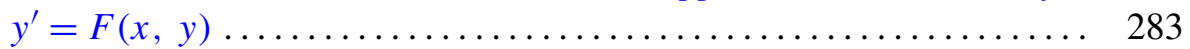

Michael Joseph Kascic, Jr., Polynomials in linear relations . . . . . . . . . . 291

Alan G. Konheim and Benjamin Weiss, A note on functions which

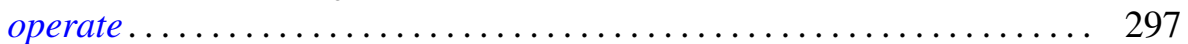

Warren Simms Loud, Self-adjoint multi-point boundary value problems ... 303 Kenneth Derwood Magill, Jr., Topological spaces determined by left ideals

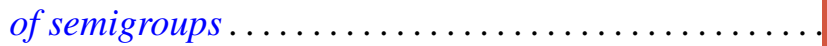

Morris Marden, On the derivative of canonical products . . .

J. L. Nelson, A stability theorem for a third order nonlinear differential

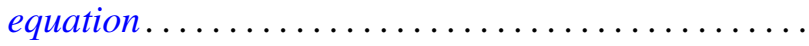

Raymond Moos Redheffer, Functions with real poles and zeros ...

Donald Zane Spicer, Group algebras of vector-valued functions ...

Myles Tierney, Some applications of a property of the functor $E f$ 